\title{
Aprendizaje en metodología de investigación para la construcción de la escala diferencial semántico apoyado con mediaciones tecnológicas
}

FECHA DE RECEPCIÓN: 17 de enero FECHA DE APROBACIÓN: 1 de febrero Pp. 117-129

\section{Resumen}

El presente artículo tiene como propósito conocer el proceso de aprendizaje en metodología de la investigación tras la práctica pedagógica en el aula, donde se amplían los conocimientos teóricos-prácticos para el diseño y construcción de la escala diferencial semántico. Este proceso se desarrolla con una metodología participativa bajo la estrategia pedagógica colaborativa, implementada con estudiantes de VIII semestre de trabajo social de la Universidad de la Guajira en el año 2015. Se apoyan los actos pedagógicos con mediaciones tecnológicas, como Cmaptools, Edraw MindMap y Prezi, además del software estadístico SPSS18. La experiencia arrojó un gran nivel de aprendizaje en cuanto a la alta competencia para definir, identificar, comparar, demostrar, diferenciar, evaluar, interpretar, seleccionar, analizar, discriminar y describir la escala; finalmente, el uso de las TIC permitió la apropiación de conceptos, teorías y postulados de investigación.

\section{Palabras clave}

Investigación, aprendizaje colaborativo, TIC, escala diferencial semántico.

\footnotetext{
Aldrin Antonio Carvajal Torres

Doctor en Investigación Científica. Magíster en Psicología Clínica y de la Salud. Maestrante Pedagogía de las TIC. Profesor de la Universidad de la Guajira, programa de Trabajo Social.
}

Nohemy De la Torre, $F$.

Magíster en Desarrollo Social, Maestrante en Pedagogía de las TIC. Profesor de la Universidad de la Guajira, programa de Trabajo Social. 


\title{
Methodological Research Learning to Build up a Semantic Differential Scale Supported by ICT Mediation
}

\begin{abstract}
Abstrac. This article aims at describing the methodological learning process of research as classroom practice allows to widen the theoretical and practical knowledge to design and build up a semantic differential scale. This process is developed based on a participatory method through a pedagogical collaborative strategy applied with VIII semester students from Social work studies at "Universidad de la Guajira" in 2015. Pedagogical acts are supported by ICT such as Cmaptools, Edraw MindMap, Prezi, and the SPSS18 statistical software. This experience shows a strong learning in terms of high competition to define, identify, compare, demonstrate, differentiate, assess, interpret, select, analyze, discriminate and describe the corresponding scale. Finally, the application of ICT allows the acquisition of new research concepts, theories and postulates.
\end{abstract}

Key words. Research, collaborative learning, ICT, semantic differential scale.

\section{Apprentissage de méthodologie d'investigation pour la construction d'échelle sémantique différentielle assistée par des outils technologiques}

\begin{abstract}
Resumé. Cet article a pour objectif de définir et présenter le processus d'apprentissage en méthodologie d'investigation dans la salle de classe (une fois le stage pédagogique réalisé) où les connaissances théoriques et pratiques sont amplifiées par la conception sémantique et la construction d'échelle différentielle. Ce processus a été développé grâce à une méthodologie participative et une stratégie pédagogique collaborative mise en place avec des étudiants de huitième semestre de Relations Sociales de I'Université de la Guajira en 2015. Les processus pédagogiques sont assistés par des outils technologiques tels que Cmaptools, Edraw MindMap, Prezi, ainsi qu'un logiciel statistique SPSS18. L'expérience rend compte d'un fort taux d'apprentissage des critères de compétence pour définir, identifier, comparer, démontrer, différer, évaluer, interpréter, sélectionner, analyser discriminer et décrire l'échelle. Enfin, I'usage des TIC a permis l'appropriation de concepts, de théories et de postulats de recherche.
\end{abstract}

Mots clefs. Recherche, apprentissage collaboratif, TIC, échelle sémantique différentielle.

\section{Metodologia de aprendizagem de pesquisa para a construção da escala diferencial semântica apoiada em mediações tecnológicas}

Resumo. Este artigo tem como objetivo conhecer o processo de aprendizagem em metodologia de pesquisa da prática pedagógica em sala de aula onde se ampliam os conhecimentos teóricos e práticos para a concepção e construção da escala de Diferencial de Semântica. Este processo é desenvolvido com uma metodologia participativa no âmbito da estratégia de ensino colaborativo, implementada com alunos do VIII semestre de serviço social da Universidade de La Guajira em 2015. Se apoia nos atos pedagógicos com mediações tecnológicas, como CmapTools, Edraw MindMap, Prezi, além do software estatístico SPSS18. A experiência mostra alta aprendizagem em critério de alta competência para definir, identificar, comparar, demonstrar, diferenciar, avaliar, interpretar, selecionar, analisar, discriminar e descrever a escala, finalmente, a utilização das TICs permitiu a apropriação de conceitos, teorias e postulados de pesquisa.

Palabras chave. Pesquisa. Aprendizagem colaborativa. TICS. Escala diferencial semántica. 


\section{Introducción}

E I artículo tiene como propósito conocer el proceso de aprendizaje en metodología de la investigación tras la práctica pedagógica en el aula, en donde se amplían los conocimientos teóricos-prácticos para el diseño y construcción de la escala de evaluación científica como la escala diferencial semántico

Para el desarrollo de esta ampliación y fortalecimiento de los conocimientos en metodología de la investigación, fue necesario utilizar, dentro de la estrategia colaborativa, las técnicas pedagógicas como la lluvia de ideas, la lectura o revisión de textos teóricos referentes a los contenidos de la formación y el estudio de casos.

Dentro de las capacidades intelectuales de orden superior que se referencian en la experiencia pedagógica, se tiene el análisis, la síntesis, la conceptualización, el manejo de información, el pensamiento crítico, el pensamiento sistémico, la investigación, la metacognición y pensar con información. De acuerdo con González (2002), estas capacidades cada vez van siendo más complejas en la medida en que se profundice el aprendizaje en investigación y se apropien y transfieran conocimientos, lo cual implica que la formación en investigación para las ciencias sociales y humanas, debe vigilar la forma de interrogar la realidad, desde la lógica del método científico y las técnicas de recolección de información. Para esta experiencia es fundamental la apropiación de las mediaciones tecnológicas para la formación de conceptos científicos en metodología de la investigación, por ello, se utilizan recursos educativos como Cmaptools e Edraw MindMap, herramientas que facilitan la formación de mapas y esquemas mentales en el sujeto, con un valor agregado, y es que son compartidos en la Internet de manera que otras personas pueden observar lo construido por el estudiante y pueden hacer comentarios sobre el producto de aprendizaje; este mismo valor se obtiene con las presentaciones creadas en Prezi. Además la utilización del software estadístico SPSS18 le permite al estudiante evaluar los primeros resultados de su aprendizaje en cuanto a la validez experimental de la escala.

Por consiguiente, este artículo se fundamenta en la presentación de una experiencia pedagógica basada en el aprendizaje colaborativo, en la búsqueda de que el estudiante se fortalezca y participe activamente en la construcción y generación de conocimientos en metodología de la investigación y que a su vez responda al mejoramiento de problemáticas del entorno social. 


\section{Desarrollo}

\subsection{Definición del tema o del área del problema}

La investigación institucional o significativa, a través de la cual se construye conocimiento universalmente nuevo y cuya originalidad y legitimidad puede ser reconocida por la comunidad académica, tiene que ver con las líneas de investigación global propuestas y con los contextos específicos; esta puede ser:

- Investigación básica: referida a la producción de conocimiento significativo en el contexto de un paradigma.

- Investigación de proyección: el saber se pone al servicio de instancias sociales que responden a las necesidades de su contexto. En síntesis, «las necesidades sociales deben ser convertidas en problemas académicos para que la academia pueda enfrentarlas con las herramientas que le son propias».

\section{- Investigación formativa o curricular:} involucra el proceso investigativo de manera proporcional a la evolución lineal del conocimiento y avance en el plan de estudios; es decir que está enfocada al aprendizaje del estudiante, al proceso de apropiación de saberes y procedimiento que se lleva a cabo en su formación disciplinar, así como al

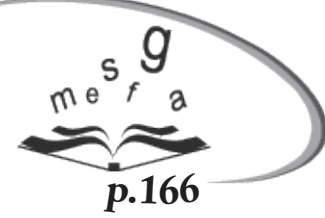
desarrollo de competencias investigativas; está destinada al conocimiento y al ejercicio del proceso investigativo; así, por medio de este tipo de investigación, el estudiante se aproxima a la disciplina y con el marco de referencia que esta le brinda, llega a desarrollar un actuar profesional, una comprensión y explicación de los fenómenos que se manifiestan en su entorno.

La formación en investigación desde el documento base de trabajo social, permite desarrollar las siguientes competencias 0 aptitudes:

- Diseñar, administrar y evaluar planes y proyectos sociales.

- Aplicar técnicas de investigación social, evaluación, sistematización y operatividad de las técnicas de intervención profesional.

- Manejar con eficiencia las técnicas de relaciones interpersonales y el diálogo como mediación pedagógica.

- Desarrollar un liderazgo proactivo y prospectivo.

- Manejar tecnologías vigentes para la recopilación, el análisis y la presentación de los hallazgos de la investigación y la práctica social.

- Integrar, con facilidad, equipos interdisciplinarios y multidisciplinarios. Se trata de la innovación, creatividad y recursividad, con el fin de establecer el equilibrio y evitar rupturas, desarraigo y violencia, para facilitar la convivencia pacífica.

El plan de desarrollo de la Universidad de 2010 - 2013, establece que el uso y apropiación de las Tecnologías de la Información y la Comunicación (TIC), garantizan el acceso, uso y apropiación critica de las TIC como herramientas para el aprendizaje, la creatividad, el avance tecnológico y cultural, y además, permiten el desarrollo humano y la participación activa en la sociedad del conocimiento. 
El informe de autoevaluación de la Facultad de ciencias sociales y humanas realizado en mayo de 2012, sobre el programa de trabajo social, orientado al documento base y en especial al área de investigación y al uso y apropiación de las TIC, refiere lo siguiente:

Que el programa de trabajo social promueve en su plan de estudio la cultura investigativa y el pensamiento crítico y autónomo en los estudiantes, sin embargo, no se definen las políticas dondese consideren los medios para que la investigación sea un compromiso en los docentes y se convierta en un reflejo de lo que los estudiantes desarrollen en su vida profesional; no define el sentido de la investigación, el alcance en la formación del estudiante, su estructura y organización; el programa no cuenta con las estrategias para incorporar el uso y apropiación de las tecnologías de la información y de la comunicación en la formación investigativa de los estudiantes (Uniguajira, 2013).

El problema en el contexto del aula, como docentes comprometidos con el proceso formativo en investigación en trabajo social, se desarrolla a partir de un ejercicio práctico en dondeel estudiante, desdela operacionalización de variables, debe hipotéticamente formular preguntas relacionadas con los indicadores de las variables, para ello debe elaborar un cuestionario cuyos Ítems tengan criterios de validez y confiabilidad, utilizando un modelo de escala tipo diferencial semántico. Una vez realizado el ejercicio dio como respuesta un desconocimiento p.166 parcial en lo teórico-conceptual, no desconociendo que el estudiante trajo consigo conocimientos adquiridos en investigación, en lo que refiere a técnicas de recolección de datos como son la entrevista, la observación, la elaboración de encuestas y el cuestionario. Lo anterior indica que sabe formular preguntas para obtener información con respecto a un tema o problema de investigación, pero también es cierto que carece de conocimientos metodológicos para la elaboración de los modelos de las escalas de evaluación.

\subsection{Conceptualización}

La formación de conceptos científicos en el sujeto, de acuerdo con Vygotsky y Piaget, bajo una óptica constructivista o cognitiva, se determina, en primera instancia, bajo estructuras mentales las cuales van dando forma a la relación que hace el sujeto de un objeto como tal representado en imágenes simbólicas, que son asimiladas, acomodadas y adaptadas en diferentes formas de organización interna denominadas en esquemas mentales, es decir, formas de organización de la información que en la vida propia del sujeto, se irán. No se pretende debatir quien tiene la razón o no, ni que teoría tiene mayor o menor importancia; tampoco, cual es más importante que otra. Solo se referenciará teóricamente cómo se da el proceso en la formación de conceptos científicos reconocidos casi que universalmente, y que para el caso de metodología de la investigación, estos son debatidos con frecuencia, ya que cada vez surgen nuevas investigaciones que hacen revaluar los procedimientos metodológicos en investigación-experimental y no experimentao al menos en una comprensión holística de la investigación. De acuerdo con los autores en mención, el conocimiento o el concepto científico es un proceso constructivo que se va formando evolutivamente en el sujeto y además está determinado por el contexto social y cultural, es decir, el medio ambiente dentro de la vida social del sujeto; comprende las premisas que se presentan más adelante. 
Para Vygotsky es fundamental la internalización:

(...) donde el conocimiento no es un objeto que se pasa de uno a otro, sino que es algo que se construye por medio de operaciones y habilidades cognoscitivas que se inducen en la interacción social, señalando que el desarrollo intelectual del individuo no puede entenderse como independiente del medio social en el que está inmersa la persona, que el desarrollo de las funciones psicológicas superiores se da primero en el plano social y después en el nivel individual. La transmisión y adquisición de conocimientos y/o patrones culturales es posible cuando de la interacción -plano interpsicológico-, se llega a la internalización -plano intrapsicológico-.

Vygotsky concebía la internalización como un proceso donde ciertos aspectos de la estructura de la actividad que se ha realizado en un plano externo, pasan a ejecutarse en un plano interno. De igual manera reconoce la acción mediada: "donde hay una aproximación cultural a la mente y comienza con el supuesto de que la acción está mediada, y que no puede ser separada del medio en el que se lleva a cabo». Es importante aclarar que la cultura es la acción mediada para la construcción del conocimiento entre la interacción social y el plano individual, además de la recolección de datos con el diseño y construcción de las escalas de evaluación en investigación, como instrumentos con los cuales el investigador puede obtener la mayor riqueza de información en su objeto de estudio (Hernández 2008; Hurtado 2010). Estos últimos autores clasifican las escalas «diferencial-semántico» como:
«Una modalidad en la cual los ítems presentados son grupos de adjetivos opuestos». Entre cada par de opuestos se ubica una línea graduada que representa un continuo de cada intensidad, en el cual el examinado deberá marcar la posición que considere conveniente en función de lo que se pregunta.

En el proceso de investigación es importante fundamentar la presencia y desarrollo de las capacidades intelectuales de orden superior, pues estas hacen referencia a aquellas potencialidades que debe tener el profesional a fin de hacer una amplia intervención investigativa de su objeto de estudio, es decir, es un abordaje pleno sobre el contexto. A partir de ello, clarifica y comprende las dinámicas intervinientes en la investigación y la estrategia de conocimiento, de tal forma que permita obtener y comprender el estudio. Estas capacidades deben estar en alerta en todo momento de la investigación y en todas las etapas o fases del proceso, teniendo en cuenta que la planeación es una determinación de la investigación que ilustra cómo será la ruta y por dónde se direccionará; también, qué se pretende en ella. La evaluación permite interpretar el desarrollo de la misma y adaptar los ajustes pertinentes. A partir de este punto, surgirá la retroalimentación del proceso con la intención de ajustar cualquier cambio, y así en vez de interferir en la investigación, esta se vea fortalecida. A continuación se presenta una descripción de los términos y conceptos de las capacidades intelectuales de orden superior pertinentes a la investigación y determinadas por González (2002); se trata de aquellas que el sujeto debe desarrollar en el transcurso de su vida, acordes con las condiciones individuales, el medio ambiente -familiar, contexto educativo- entre otros: 
- Análisis

- Síntesis

- Conceptualización

- Manejo de información.

- Pensamiento sistémico.

- Pensamiento crítico.

- Investigación

- Metacognición

Conforme al trabajo de Biggs (2006), «calidad del aprendizaje universitario», la práctica pedagógica en cualquier escenario, contexto o situación es compleja, tanto para el docente como para los estudiantes, ya que durante el desarrollo de la historia son muchos los modelos pedagógicos propuestos y son diversas las estrategias de aprendizaje que se han presentado para mejorar los procesos de formación. De igual manera, los estudios sobre estilos de enseñanza y aprendizaje con sus características; todos ellos con el objetivo de garantizar efectivamente la asimilación de los

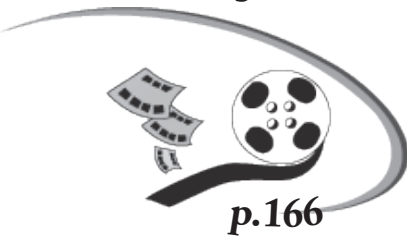
conocimientos, y sobre todo, que el estudiante los tenga presente en sus prácticas para formar a un sujeto dotado de información. En ocasiones resulta insuficiente, de acuerdo con las nuevas exigencias, que los contextos sociales, culturales, laborales académicos, entre otros, requieran que cada día se esté más y mejor preparado.

Es por ello que Biggs, en un contexto pedagógico, sugiere cuatro factores que fortalecen la adquisición del conocimiento, como son:

- Una base de conocimiento bien estructurada.

- Un contexto motivador.

- La actividad del aprendiz.

- La interacción con los demás.
Estos factores siempre están presentes en todo acto pedagógico, independientemente del enfoque o modelo pedagógico. Se deben tener en cuenta con el fin de que el proceso de formación en el estudiante sea consistente. Seguramente existirán otros factores asociados a los anteriores, que también incidirán positiva o negativamente en el aprendizaje. De igual manera y siguiendo al autor, la estructura del conocimiento estaría determinada por contenidos o conceptos que demarcan el esquema central del proceso de formación; además, estos tienen subtemas que son subordinados con la intensión de ir organizando cada parte del mismo e ir clarificando cada elemento, el cual dará una forma amplia y concreta a los conceptos a aprender. Con respecto al tema motivador es indudable reconocer, que tanto los estudiantes como el profesor, deben tener, a partir del interés, un sentido por lo que están haciendo, por lo que están construyendo, y por lo que se está impartiendo y recibiendo, de manera que esta condición del proceso psicológico sea una base sólida y determinante para el estudiante en particular; es decir, se aprende del error y de las expectativas de aprendizaje. Luego, se hacen proyecciones y proponen soluciones diversas a problemas planteados en clase o en diferentes contextos.

Ahora, con respecto a las actividades de aprendizaje, es claro que el buen desarrollo de las mismas estarán de forma interactiva, refiriéndose a la participación activa dirigida y autodirigida por los estudiantes y el profesor, siendo este último el facilitador y mediador de los proceso de formación conceptual. Por esta razón, en el desarrollo del proyecto de profundización, el estudiante tiene una base de textos sobre temas estructurados a partir de los cuales, él se prepara para socializarlos a los compañeros a través de herramientas como Prezi, además de la construcción de mapas 
conceptuales elaborados con Edraw MindMap y Cmaptools, cuya actividad será orientada por el docente. Así mismo, los mapas conceptuales presentan un esquema general y cada vez más preciso sobre la comprensión holística de un tema en particular, que deter-minan toda las complejidades de los conceptos y que son útiles en la presentación del desarrollo de las habilidades de orden superior.

Con respecto al conocimiento y su formación en el contexto universitario, es preciso dar a conocer que de acuerdo con Biggs, este en primera instancia, es de tipo declarativo, abstracto y especialmente conceptual; además, su cometido consiste en nombrar, diferenciar, desarrollar y justificar. En segunda instancia, el conocimiento profesional es de tipo procedimental, específico y pragmático. Por ello, su contenido consiste en ejecutar, aplicar y establecer prioridades. Para efecto del proceso de formación investigativa, no cabe la menor duda de que la formación estará orientada a la conceptualización, a la declaración y lo abstracto, pues ese sería su fuerte y su razón de ser; se sabe que el futuro profesional que va a estar en el campo laboral, se debe ir fortalecido con unas bases teóricas que le permitan analizar cualquier escenario, que a partir de allí, formule proyectos de investigación o de intervención con miras a conocer las problemáticas y resolver situaciones.

Los autores Cañas, Ford, Hayes, Reichherzer, Suri, Coffey, Carff y Hill, sugieren que con respecto a los procesos de construcción del conocimiento con el uso de los mapas conceptuales y los conceptos desarrollados por Novak (1977), se aproveche el uso del lenguaje para la descripción y comunicación de conceptos dentro de la teoría de asimilación. Se trata de una teoría del aprendizaje que ha tenido una enorme influencia en la educación (Ausubel et al., 1978): está basada en un modelo constructivista de los procesos cognitivos humanos. En particular, la teoría de asimilación describe cómo el estudiante adquiere conceptos y cómo se organiza en su estructura cognitiva. Es decir, los mapas conceptuales son una técnica pedagógica que permite, a través de la construcción de diferentes representaciones gráficas, los conceptos, de manera que las interrelaciones establecidas entre cada uno sean notorios o visualizados fácilmente pues estas terminan cada una con frases simplificadas. El proceso de construcción del mapa mental requiere, dentro de las habilidades de orden superior análisis, síntesis, conceptualización, manejo de información, pensamiento sistémico, pensamiento crítico, investigación, metacognición y pensar con información; pero independiente de todas estas habilidades, el mapa mental lo puede representar tanto los niños como los adultos. Por su parte, el mapa conceptual puede ser sencillo como también complejo, es decir, que teniendo múltiples enlaces, tendría múltiples conectores; además, con el apoyo de las mediaciones tecnológicas, los software de los mapas mentales con base en los Recursos Educativos Abiertos (REA), son conocidos: Cmaptools e Edraw MindMap. Estos son instalados en los ordenadores y permiten construir conceptos desde el mismo PC conectados a Internet o sin conexión; están dotados de múltiples herramientas, de manera que permiten el ingreso de textos, imágenes, videos, audios, etc.

Según Aguilar (2006), el mapa conceptual se convierte en una herramienta para aprender y actuar, pues esta es una técnica muy útil para el aprendizaje colaborativo que además estimula la reflexión en grupos de aprendizajes y también en las organizaciones. Como principio a tener en cuenta, se construye a partir de conceptos ordenados jerárquicamente, y 
en aquellos de mayor generalidad, ocuparán espacios superiores. Como herramienta pedagógica se pueden utilizar a partir del desarrollo de temas o notas de clase, y para ordenar y representar los conocimientos y teorías. Finalmente, Aguilar concluye que los mapas mentales dentro de las funciones más importantes que cumplen en el proceso de la construcción del conocimiento, está la de definir la formación de un esquema general del tema a desarrollar en una clase o curso de formación; también es una herramienta de diagnóstico que le permite al profesor conocer los conocimientos que el alumno tiene con respecto a un tema, y de esta forma, planificar la estrategia de instrucción. El mapa conceptual es un verdadero instrumento de evaluación que permite observar el cambio en las estructuras cognitivas de los estudiantes; es una estrategia dinámica grupal para la participación e interacción de significados de conceptos de doble vía -profesor-alumno-; así mismo, es útil para la organización, planeación y desarrollo de la clase.

La obra de Castañeda, Cabrera, Navarro y De Vries (2010), «Procesamiento de datos y análisis estadísticos utilizando SPSS», permite que con el SPSS -software estadístico con aplicaciones prácticas para las investigaciones, especialmente las de carácter social-, se establezcan vínculos con otros programas como son Microsoft Word, Microsoft Excel, y se realicen análisis estadísticos básicos, inclusive muy complejos. Los autores en mención, analizan las ventajas de SPSS como aquellos diferentes análisis estadísticos descriptivos que permiten profundizar en las variaciones que se presentan en una investigación.

Escalante (2011), en «SPSS- 5 procesos básicos», determina que los cinco procesos básicos que debería realizar un investigador o tesista con sus datos deben ser: explorar, frecuencia, descriptivo, tabla de contingencia y correlación. Es un video de la categoría de formación educativa, el cual orienta paso a paso la aplicación del software en la investigación social.

Sánchez, Pardo e Izquierdo (2010), argumentan que en el proceso de formación para la investigación científica con el empleo de las TIC, la apropiación del contenido tecnoinvestigativo abarca aspectos tecnológicos y de la investigación científica con respecto al desarrollo de las habilidades básicas en el manejo de la información -navegar e interactuar-, en el procesamiento de la documentación digital, el dominio de un grupo de herramientas generales relativas a la búsqueda, revisión, procesamiento y comunicación de la información digital, de manera que lo tecnológico sustenta a las TIC y está referido a todas las aportaciones asociadas a estas tecnologías. Por su parte, lo investigativo subyace en la educación superior a partir de la construcción de los contenidos científicos, lo que está en la propia esencia del proceso docente educativo universitario.

Colas y De Pablos (2012), sostienen que la TIC se presentan como potentes herramientas con la capacidad de mejorar considerablemente la calidad de la investigación cualitativa, teniendo impacto en todos los criterios de calidad tradicionalmente utilizados en dicha investigación es decir, credibilidad, transferibilidad, dependencia y confiabilidad. Las TIC facilitan y mejoran los procedimientosqueseaplican en la metodología cualitativa para asegurar su calidad científica, tales como recogida mecánica de abundantes datos y contrastación de los resultados. Son fundamentales en la determinación de los muestreos, en la recogida de datos y en el análisis de la información. 
Nieto y Rodríguez (2007) sugieren que un aprendizaje de alto nivel precise una intensa actividad intelectual y facilite la comprensión profunda, ya que dota al análisis de recursos que favorecen estructuras cognoscitivas complejas, exige una gran variedad y riqueza de actividades intelectuales, así como un uso estratégico de procedimientos cognitivos progresivamente más complejos y refinados. Por ello, en la actividad pedagógica el docente utiliza diferentes estrategias con el fin de que los estudiantes asimilen los conocimientos, se fortalezcan en la medida en que la innovación pedagógica sea más participativa y demande mayor activación en las personas. Esa innovación, acompañada de recursos tecnológicos, facilita la construcción de conocimiento. Estos mismos autores consideran que la actividad pedagógica, en cuanto a la conceptualización de los diseños de investigación, genera el planteamiento de cuestiones significativas y relevantes que pueden ser investigadas de forma empírica como: ligar la investigación a una teoría relevante; proporcionar una cadena de razonamiento manifiesta y coherente; distinguir métodos y datos para facilitar y estimular el escrutinio y crítica del trabajo e intentar resultados que puedan replicarse y generalizarse.

\section{Propósito}

\subsection{Desde lo general}

Ampliar los conocimientos teórico-prácticos en metodología de la investigación para el diseño y construcción de las escalas de evaluación científica como escala diferencial semántico.

\subsubsection{Desde lo específico centrado en el aprendizaje}

Aprender a identificar colaborativamente criterios que definan una investigación, como son: definir, clasificar, caracterizar, identificar, comparar, demostrar, diferenciar, evaluar, interpretar, seleccionar, analizar, discriminar, describir, debatir, proponer y compartir información, para un aprendizaje significativo de la escala diferencial semántico apoyado en las herramientas tecnológicas apropiadas para la investigación

\subsection{Ejecución: objetivos de aprendizaje}

\subsubsection{Estrategia pedagógica por etapas}

Con relación a la pertinencia en desarrollo de una metodología estratégica colaborativa, se determinaron tres etapas del proceso pedagógico que a continuación se describen:

\section{- Capacitación para el desarrollo de competencias tecnológicas}

En esta etapa se capacitó al estudiante sobre el uso y aplicación de las mediaciones tecnológicas que son apoyo para la formación en investigación: Edraw MindMap, Cmaptools y Prezi; y el software estadístico SPSS18 (Figura 1). 
Figura 1. Capacitación tecnológica

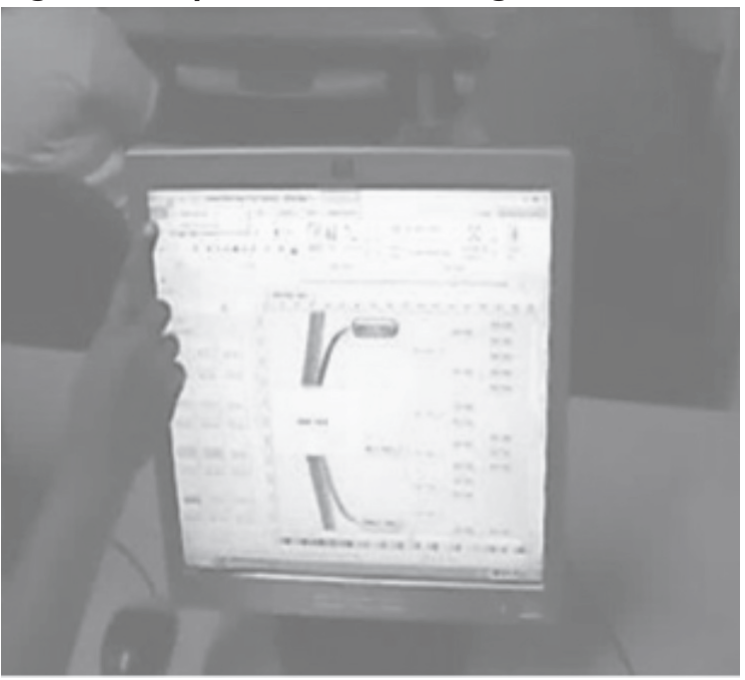

Fuente. Elaboración propia de los autores, (s.f)

\section{- Formación conceptual en metodología de la investigación}

Se desarrolló en seis sesiones con temas relacionados a la investigación, es decir, hubo recolección de datos y diseño de escalas de evaluación en la investigación social y el análisis de información. En este proceso se distribuyeron los temas y subtemas entre ocho grupos de trabajo bajo el siguiente orden:

- Recolección de datos con los principios y evidencias metodológicas y científicas para la construcción de un instrumento de evaluación.

- Fases y procedimientos para la construcción de un instrumento o escalas de evaluación.

- Conocimiento sobre la escala de diferencial semántico.
- Proceso cognoscitivo en capacidades intelectuales de orden superior para el análisis e interpretación de resultados de la escala de evaluación.

Figura 2. Socialización del conocimiento.

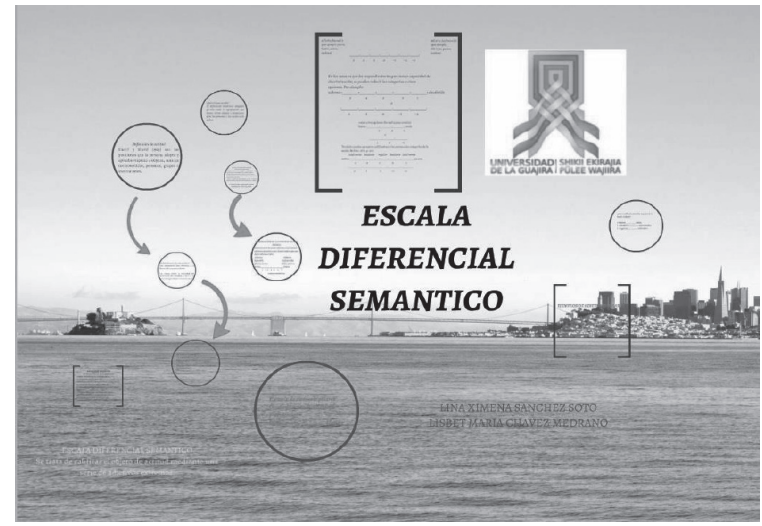

Fuente. Elaboración propia de los autores, (s.f).

- Contextualización práctica: se trata de llevar la teoría a realidades prácticas en la aplicación del conocimiento

En ese orden de ideas y teniendo claridad en el tema seleccionado, se identificaron los contextos objeto de estudio con características similares, como fue el municipio de El Molino, ubicado al sur del departamento de la Guajira y que ha atravesado problemas de orden público influenciados por la incursión de grupos al margen de la ley. Lo anterior indica un alto grado de violencia e inseguridad, razón por la cual se diseñó la escala y se aplicó una prueba piloto con el tema sobre violencia social.

Posterior a la aplicación de la prueba piloto, se procedió a evaluar los resultados teniendo en cuenta el aprendizaje propuesto y la estrategia pedagógica. 


\section{Resultados}

E I presente análisis de resultados hace parte de la experiencia académica en desarrollo de una estrategia pedagógica colaborativa, la cual se construyó, amplió y fortaleció en el aprendizaje, y por consiguiente, los conocimientos en metodología de la investigación, utilizados con diferentes técnicas pedagógicas didácticas -lecturas autorreguladas, Iluvia de ideas, debates, estudio de casos, construcción de mapas conceptuales-, apoyados en mediaciones tecnológicas que hicieron que cada acto pedagógico fuera un momento único y significativo en la formación profesional del estudiante de la asignatura electiva de investigación.

En cuanto al aprendizaje en metodología de investigación para la construcción de la escala diferencial semántico, apoyado con mediaciones tecnológicas y utilizando esta estrategia pedagógica colaborativa, se puede afirmar que los estudiantes alcanzaron una alta competencia en habilidades de pensamiento de orden superior, las cuales se fueron desarrollando en actividades teórico-prácticas que determinaron la formación conceptual. En lo referente al aprendizaje de la escala diferencial semántico, arrojó como resultado la apropiación teórico-práctica de la mismas, lo que pronostica una buena producción investigativa a futuro, con confiabilidad y validez científica en los instrumentos diseñados.

Analizando los resultados que referencian el aprendizaje del análisis e interpretación de resultados de investigación como una cualificación de la formación investigativa, se considera que los estudiantes desarrollaron habilidades de pensamiento de orden superior orientadas en competencias, que permitieron aplicar los conocimientos adquiridos en el desarrollo de la formación investigativa.

De otra parte y con relación a los planteamientos básicos sugeridos por Biggs para que se fortalezcan los conocimientos, es fundamental el hecho de partir de una base de conocimientos estructurados, es decir, organizar los contenidos y temas teóricos que serán abordados en el aula. Así, los estudiantes hacen un discernimiento de cada concepto y teoría abordada, lo que permite desarrollar un pensamiento crítico sobre los documentos. Otro aspecto a resaltar está en la creación del contexto motivador, es decir, un ambiente favorable que invite al estudiante a participar, a ser activo, a expresar sus puntos de vista. Con respecto a las actividades del aprendiz, es claro que una vez se tienen presente las anteriores, se prosigue a definir cuáles son las actividades de aprendizaje del estudiante y las acciones encaminadas a la ejecución del conocimiento; en este aspecto, el aprendizaje colaborativo toma importancia porque es el momento donde responde a cada trabajo. Es vital tener en cuenta que la interacción con los demás, como última premisa que sugiere Biggs, en cuanto al aprendizaje colaborativo, es que comparta con otros su conocimiento y de igual manera sus dudas, para ser resueltas entre sus compañeros. 


\section{Referencias bibliográficas}

Aguilar. M. (2006). El mapa conceptual una herramienta para aprender y enseñar. Plasticidad y Restauración Neurológica, 5(1). Recuperado de: http://bit.ly/1xMdZJd.

Bernal, C. A. (2010). Metodología de la investigación. Administración, economía, humanidades y ciencias sociales. Bogotá: Pearson, Tercera edición.

Biggs. J. (2006). Calidad del aprendizaje universitario. Recuperado de: http://bit. ly/1V2YJKP.

Castañeda. M, Cabrera. A, Navarro. Y y De Vries, W. (2010). Procesamiento de datos y análisis estadísticos utilizando SPSS. Un libro práctico para investigadores educativos. Brasil: EDIPUCRS, recuperado de: http:// www.pucrs.br/edipucrs/spss.pdf

Cañas, A, Ford. K, Hayes. P, Reichherzer. T, Suri. N, Coffey. J, Carff. R, Hill.G. Sin Título. Recuperado de: http://www.ihmc.us/

Colas, P y De Pablos J. (2012). Sin título. http:// bit.ly/14eYKQ2.

Escalante. E. (2011). SPSS- 5 Procesos Básicos. Youtube. Recuperado de: http://bit. ly/1sDm3sb.

González, H. Z. (2002). Capacidades de pensamiento de orden superior. Edukateka. Recuperado de: http://www.eduteka.org/ CapacidadesMentales.php
Hernández, S.R, Fernández, C.C y Baptista, L.P. (2010). Metodología de la investigación México: McGraw-Hill Interamericana, quinta edición.

Hurtado, J. (2011). Metodología de la Investigación, hacia la comprensión holística de la Ciencia. (s.c.): Editorial Quirós, cuarta edición.

Muhammad, N. N., (2005). Metodología de la investigación. (s.c.): Limusa Editores, segunda edición.

Nieto, S. y Rodríguez, M. (2007). Convergencia de resultados en dos diseños de investigación-innovación en enseñanza universitaria a través de las TIC. Revista Española de Pedagogía. LXV(236), 27-48. Recuperado de: http://bit.ly/11hsuKQ.

Sánchez, L., Pardo, M. e Izquierdo, J. (2010). El proceso de formación para la investigación científica en la educación superior sustentado en las tecnologías de la información y las comunicaciones. Santiago de Cuba. Recuperado de: http://www.redalyc. org/pdf/1813/181317848011.pdf

Universidad de la Guajira (2013). Plan de Desarrollo (2010-2013). Riohacha: Uniguajira. Recuperado de: http://www. uniguajira.edu.co. 